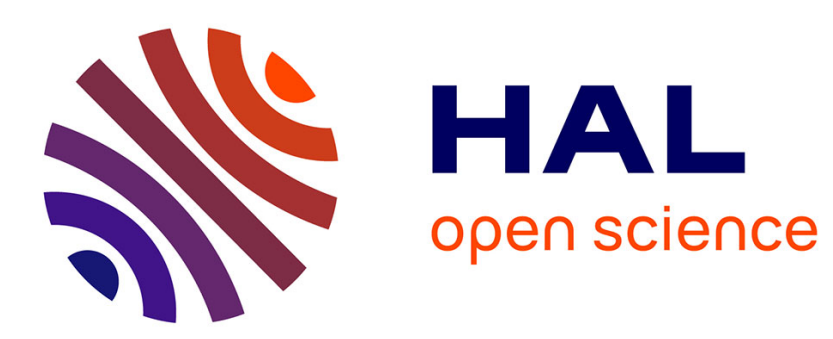

\title{
Anisotropie de l'atténuation ultrasonore au-dessus d'une transition smectique a-nématique. Détermination d'un temps de relaxation
}

\author{
J.C. Bacri
}

\section{- To cite this version:}

J.C. Bacri. Anisotropie de l'atténuation ultrasonore au-dessus d'une transition smectique a-nématique. Détermination d'un temps de relaxation. Journal de Physique Lettres, 1975, 36 (6), pp.177-180. 10.1051/jphyslet:01975003606017700 . jpa-00231182

\section{HAL Id: jpa-00231182 https://hal.science/jpa-00231182}

Submitted on 1 Jan 1975

HAL is a multi-disciplinary open access archive for the deposit and dissemination of scientific research documents, whether they are published or not. The documents may come from teaching and research institutions in France or abroad, or from public or private research centers.
L'archive ouverte pluridisciplinaire HAL, est destinée au dépôt et à la diffusion de documents scientifiques de niveau recherche, publiés ou non, émanant des établissements d'enseignement et de recherche français ou étrangers, des laboratoires publics ou privés. 


\title{
ANISOTROPIE DE L'ATTÉNUATION ULTRASONORE AU-DESSUS D'UNE TRANSITION SMECTIQUE A-NÉMATIQUE. DÉTERMINATION D'UN TEMPS DE RELAXATION
}

\author{
J. C. BACRI
}

Laboratoire d'Ultrasons $\left({ }^{*}\right)$, Université Paris VI, Tour 13, 4, place Jussieu, 75230 Paris-Cedex 05, France

(Reçu le 4 février 1975, révisé le 21 mars 1975, accepté le 9 avril 1975)

\begin{abstract}
Résumé. - Nous avons mesuré l'anisotropie d'atténuation ultrasonore dans la phase nématique d'un cristal liquide au voisinage d'une transition smectique A-nématique faiblement du premier ordre, à trois fréquences $220 \mathrm{MHz}, 560 \mathrm{MHz}$ et $840 \mathrm{MHz}$. Nous avons déterminé un temps de relaxation $\tau=\tau_{0}\left(\Delta T / T_{\mathrm{c}}\right)^{-1}$ avec $\tau_{0}=3 \times 10^{-12} \mathrm{~s}$.
\end{abstract}

\begin{abstract}
We have measured the anisotropic ultrasonic attenuation in the nematic phase near a weakly first order nematic-smectic A phase transition at three frequencies $220 \mathrm{MHz}, 560 \mathrm{MHz}$ and $840 \mathrm{MHz}$. We have determined a relaxation time $\tau=\tau_{0}\left(\Delta T / T_{\mathrm{c}}\right)^{-1}$ with $\tau_{0}=3 \times 10^{-12} \mathrm{~s}$.
\end{abstract}

1. Introduction. - De Gennes a montré que pour une transition smectique A-nématique on pouvait développer l'énergie libre $F$ en fonction d'un paramètre d'ordre complexe $\psi=\psi_{0} \mathrm{e}^{i \varphi}$ où $\psi_{0}$ représente la densité des couches et $\varphi$ repère leur position [1]. $\mathrm{Au}$ voisinage de $T_{\mathrm{c}}, \psi$ est une fonction lentement variable de $F$ et donne une approximation de champ moyen :

$$
\begin{aligned}
F=A|\psi|^{2} & +\frac{1}{2} B|\psi|^{4}+\frac{1}{2 M_{\mathrm{V}}}\left|\nabla_{\mathrm{Z}} \psi\right|^{2} \\
& +\frac{1}{2 M_{\mathrm{T}}}\left|\left(\nabla_{\perp}-i q_{\mathrm{s}} \delta \mathbf{n}_{\perp}\right) \psi\right|^{2}+F_{\mathrm{el}}
\end{aligned}
$$

où $M_{\mathrm{V}}, M_{\mathrm{T}}$ sont les valeurs principales du tenseur de masse $M$, le long de l'axe et perpendiculairement à l'axe et où $F_{\text {el }}$ est l'énergie élastique de Frank.

La minimisation de cette énergie libre de type Landau conduit d'abord à un comportement divergent des constantes de Frank $K_{22}$ et $K_{33}$ tel que

$$
\delta \tilde{K} \sim \xi(T)=\xi_{0}\left[\frac{T-T_{\mathrm{c}}}{T_{\mathrm{c}}}\right]^{0,66}
$$

$\xi(T)$, longueur de cohérence.

D'autre part, F. Brochard et F. Jähnig [2] en utilisant ici encore l'analogie avec la transition $\lambda$ de l'hélium, ont constaté que certains coefficients de viscosité comportaient eux aussi une partie critique

$$
\delta \tilde{\eta}(\omega)=\frac{\delta \tilde{\eta}(0)}{1+\omega^{2} \tau^{2}} \quad \text { avec } \quad \delta \tilde{\eta}(0) \sim \frac{\tau(T)}{\xi(T)}
$$

(*) Equipe de Recherche Associée au C.N.R.S. où

$$
\tau(T) \sim \xi^{3 / 2}(T)
$$

est le temps de relaxation du paramètre d'ordre.

Expérimentalement, $\delta \widetilde{K}_{22}$ et $\delta \widetilde{K}_{33}$ ont été observés $[3,4,5,6,7]$ ainsi que $\delta \tilde{\gamma}$, coefficient de friction $[8,9,10,11]$. En mesurant l'anisotropie de l'absorption ultrasonore dans la phase nématique dans le BBOA $\left({ }^{1}\right)$ à plusieurs fréquences, nous avons été en mesure d'accéder à d'autres coefficients de viscosité critiques et de mesurer le temps de relaxation $\tau(T)$ du paramètre d'ordre; ce sont ces expériences que nous décrivons dans cette lettre.

2. Méthode expérimentale. - La méthode a déjà été présentée [12]. L'appareillage est un système classique d'impulsions utilisé en transmission. Les transducteurs ultrasonores employés sont des barreaux de quartz en coupe $X$. Le cristal liquide est déposé entre les deux quartz préalablement traités pour obtenir, en l'absence de champ magnétique, le directeur parallèle au vecteur d'onde ultrasonore. Les épaisseurs sont déterminées par une cale en aluminium respectivement de $140 \mu, 70 \mu$ et $40 \mu$ pour des expériences à $220 \mathrm{MHz}, 560 \mathrm{MHz}$ et $820 \mathrm{MHz}$. La température de l'ensemble est régulée à $0,02^{\circ} \mathrm{C}$. La mesure de l'intensité du faisceau ultrasonore transmis en fonction de l'orientation du champ magnétique appliqué donne la variation de l'atténuation ultrasonore avec l'angle $\theta$ que font le vecteur d'onde et le directeur. En l'absence de champ magnétique, on mesure $\alpha_{\|}$(directeur parallèle au vecteur d'onde). Avec un fort champ magnétique (12000 G) perpendiculaire au vecteur d'onde, on mesure $\alpha_{\perp}$. 
La différence d'atténuation $\Delta \alpha=\alpha_{\|}-\alpha_{\perp}$ est proportionnelle à la combinaison linéaire suivante des coefficients de viscosité [12]

$$
\Delta \alpha \simeq \eta_{1}-\eta_{2}-\eta_{4} .
$$

Le cristal liquide utilisé est le BBOA ( ${ }^{1}$ ) (Butyloxybenzylidène octylaniline) qui a une transition de phase smectique A-nématique à $63^{\circ} \mathrm{C}$ avec une faible chaleur de transition $(80 \mathrm{cal} / \mathrm{mole})$.

3. Résultats expérimentaux. Interprétation. - Nous avons étudié uniquement l'anisotropie d'atténuation $\Delta \alpha$; on élimine ainsi une grande partie du fond continu qui n'a pas d'intérêt ici.

Contrairement aux résultats obtenus avec le CBOOA [13], on n'observe pas dans BBOA $\left(^{1}\right)$ de divergence de $\Delta \alpha$ au voisinage de la transition. C'est d'ailleurs ce qui a motivé notre recherche. A $220 \mathrm{MHz}$, on observe au contraire un maximum d'atténuation qui a lieu pour $\Delta T=4 \pm 1^{\circ} \mathrm{C}$. Ce maximum se déplace vers les hautes températures à $560 \mathrm{MHz}$ $\left(\Delta T=7 \pm 1^{\circ} \mathrm{C}\right.$ ) et disparaît à $820 \mathrm{MHz}$ (voir Fig. 1, 2 et 3).

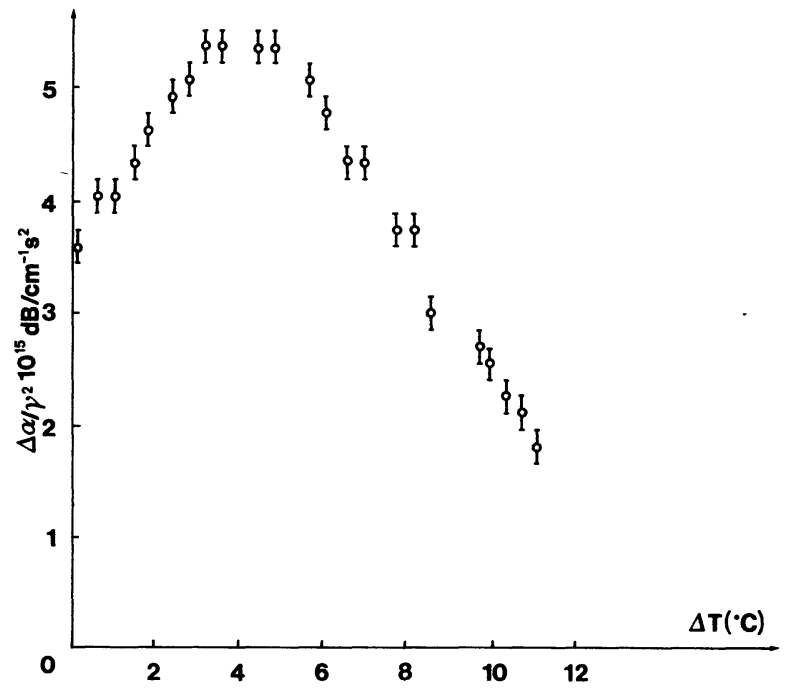

Fig. 1. - Anisotropie de l'atténuation ultrasonore à $220 \mathrm{MHz}$ en fonction de la température.

On peut rendre compte de ces résultats de la manière suivante.

Dans le cas d'un système compressible

$$
\frac{\Delta \alpha}{v^{2}} \frac{\alpha_{\|}-\alpha_{\perp}}{v^{2}} \sim \eta_{1}^{\mathrm{MPP}}-\eta_{2}^{\mathrm{MPP}}-\eta_{4}^{\mathrm{MPP}} .
$$

D'après F. Jähnig [15], on sait que $\eta_{1}^{\text {MPP }}$ diverge, que $\eta_{4}^{\mathrm{MPP}}$ diverge faiblement car il dépend d'un coefficient

( $\left.{ }^{1}\right)$ Ce composé a été synthétisé dans le Laboratoire de Physique des Solides à Orsay par L. Liebert et L. Strezelecki.

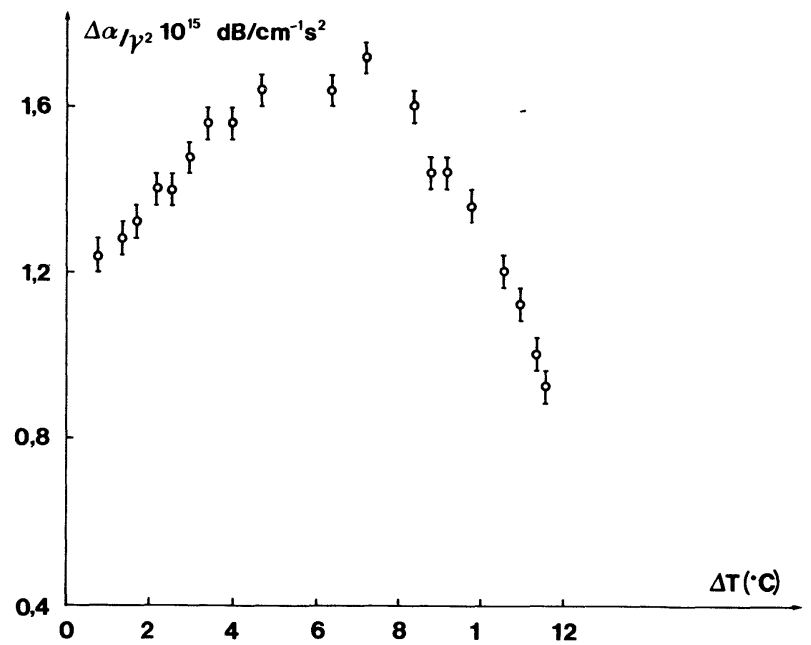

FIG. 2. - Anisotropie de l'atténuation ultrasonore à $560 \mathrm{MHz}$ en fonction de la température.

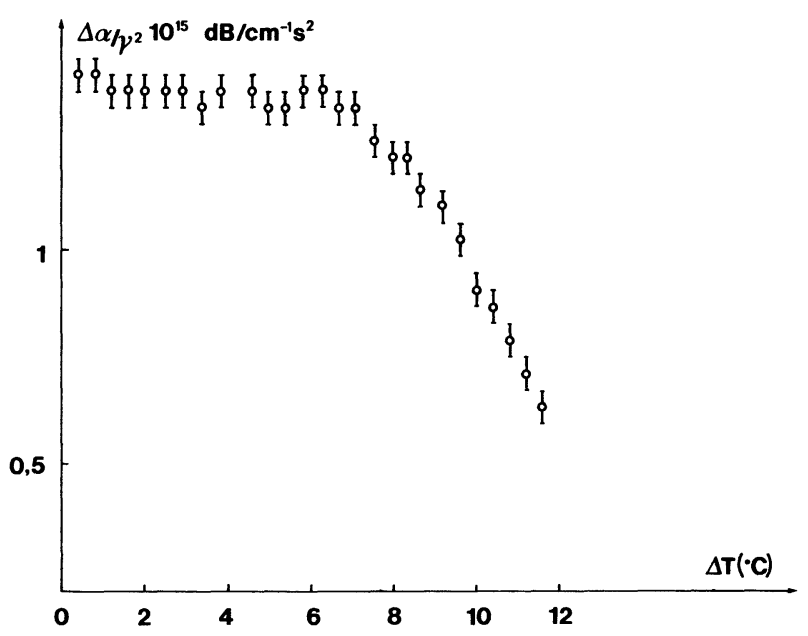

Fig. 3. - Anisotropie de l'atténuation ultrasonore à $840 \mathrm{MHz}$ en fonction de la température.

de couplage entre le paramètre d'ordre et la dilatation qui est petit, et que $\eta_{2}$ ne diverge pas. On a

$$
\delta \tilde{\eta}_{1}^{\mathrm{MPP}}(0)=\frac{M_{\mathrm{T}}}{M_{\mathrm{v}}} \frac{T q_{\mathrm{s}}^{2} \tau}{16 \pi \xi_{\|}}
$$

En combinant les formules (3), (5) et (6), on obtient donc que la partie critique de l'anisotropie d'atténuation $\Delta \tilde{\alpha}$ est

$$
\frac{\Delta \tilde{\alpha}}{v^{2}} \sim \frac{\delta \tilde{\eta}_{1}(0)}{1+\omega^{2} \tau^{2}} \sim \frac{\tau(T)}{\xi(T)} \frac{1}{1+\omega^{2} \tau^{2}(T)}
$$

$\xi$ et $\tau$ sont définis en fonction de $\Delta T$ par (2) et (4).

et

$$
\xi(T)=\xi_{\|}(T)=\xi_{0 \|}\left[\frac{\Delta T}{T_{\mathrm{c}}}\right]^{-0,66}
$$

$$
\tau(T)=\tau_{0}\left[\frac{\Delta T}{T_{\mathrm{c}}}\right]^{-1} \text {. }
$$


Sur la figurè 4 nous avons porté $\Delta \alpha / v^{2}$ en fonction de $\Delta T$ aux trois fréquences étudiées ainsi que les courbes théoriques correspondantes d'après l'expression (7).

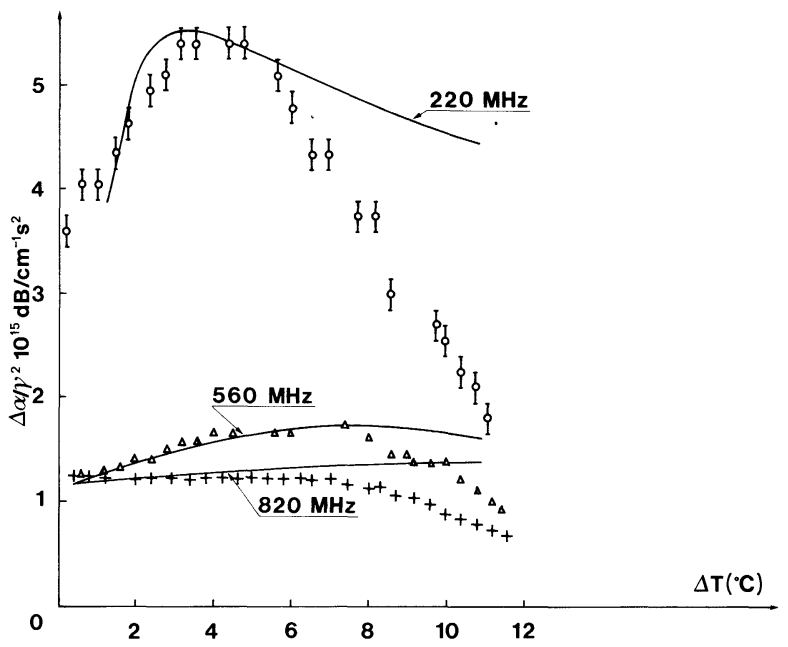

Fig. 4. - Anisotropie de l'atténuation ultrasonore, courbes expérimentales et théoriques d'après l'expression (7).

Le meilleur accord est donné pour

$$
\tau_{0}=(3 \pm 1) \times 10^{-12} \mathrm{~s} .
$$

Sur les courbes théoriques les maxima ont lieu à $3{ }^{\circ} \mathrm{C}$, $7,6^{\circ} \mathrm{C}$ et $11,2{ }^{\circ} \mathrm{C}$ pour $220 \mathrm{MHz}, 560 \mathrm{MHz}$ et $820 \mathrm{MHz}$.

A $820 \mathrm{MHz}$, le maximum théorique se trouve reporté au voisinage de la transition nématique-liquide isotrope ; c'est pourquoi on ne distingue pas de maximum sur la courbe expérimentale.

La concordance entre les résultats expérimentaux et les prévisions théoriques est bonne à condition de se trouver loin de la transition nématique-liquide isotrope. Il faut souligner qu'on ne dispose que d'une seule constante ajustable pour cet ensemble de courbes : c'est $\tau_{0}$.

L'expression exacte de $\Delta \tilde{\alpha} / v^{2}$ d'après (2) s'écrit :

$$
\frac{\Delta \tilde{\alpha}}{v^{2}}=\frac{2 \pi^{2}}{\rho v^{3}} \frac{M_{\mathrm{T}}}{M_{\mathrm{v}}} \frac{K T q_{\mathrm{s}}^{2} \tau_{0}}{16 \pi \xi_{0}} \frac{\left(\Delta T / T_{\mathrm{c}}\right)^{-0,33}}{1+\omega^{2} \tau^{2}}
$$

Pour $v=220 \mathrm{MHz}$, on trouve comme valeur théorique pour le maximum d'atténuation :

$$
\frac{\Delta \tilde{\alpha}_{M}}{v^{2}}=7 \times 10^{-15} \mathrm{~dB} \mathrm{~cm}^{-1} \mathrm{~s}^{2} \quad(\omega=2 \pi v) .
$$

La valeur expérimentale trouvée est

$$
\frac{\Delta \tilde{\alpha}_{M}}{v^{2}}=5 \times 10^{-15} \mathrm{~dB} \mathrm{~cm}^{-1} \mathrm{~s}^{2} ;
$$

elle est très proche de la valeur théorique.
Quand on se trouve au voisinage de $T_{\mathrm{c}}, \Delta \alpha$ est proportionnel à $\tilde{\eta}_{1}\left(\tilde{\eta}_{1} \gg \eta_{1}-\eta_{2}-\eta_{4}\right)$, loin de $T_{\mathrm{c}}$, $\widetilde{\eta}_{1}$ est petit et $\Delta \alpha$ est proportionnel à $\eta_{1}-\eta_{2}-\eta_{4}$. Ceci peut nous fournir l'explication du désaccord entre les courbes théoriques et expérimentales quand $\Delta T$ est grand.

L'anisotropie de l'atténuation ultrasonore en fonction de la température nous montre clairement que nous sommes en présence d'un processus à temps de relaxation non hydrodynamique variant en $(\Delta T / T)^{-1}$, mais nous ne pouvons assurer que ce temps de relaxation soit celui du paramètre d'ordre.

4. Conclusion. - Contrairement au CBOOA, nous n'avons pas pu estimer d'exposant critique pour $\eta_{1}$ dans le BBOA parce que $\tau_{0}$ est ici dix fois plus grand. Pour des basses fréquences acoustiques, on pourrait espérer faire apparaître ce comportement critique, mais on se heurte à la difficulté de préparer des échantillons monodomaines dans des volumes relativement grands. Inversement, pour des hautes fréquences, la partie critique de $\Delta \tilde{\alpha}$, en raison du dénominateur de (8), devient petite par rapport au fond continu d'atténuation qui croît en $\omega^{2}$. C'est la sensibilité des mesures qui limite l'extension de ces expériences vers les hautes fréquences. L'intérêt des mesures que nous avons effectuées ici réside dans la détermination de $\tau_{0}$ indépendamment de tout autre paramètre, ce qui s'avère impossible dans CBOOA par exemple.

\section{Nous voudrions ajouter deux commentaires :}

- La forme (3) de la viscosité est typique de celle qu'on appelle de Maxwell en théorie des liquides simples où $\tau$ est alors un temps de relaxation caractéristique d'un degré de liberté supplémentaire (collision). (3) assure que le moment d'ordre deux de la fonction de corrélation du courant est fini. Mais (3) n'assure pas que les autres moments le soient; en principe (3) n'est donc qu'une première estimation. Comme nos résultats expérimentaux ne sont pas précis au point de devoir modifier (3), nous nous sommes contentés de cette expression.

- Le comportement critique de l'atténuation que nous observons ići est caractéristique d'un processus de relaxation de Landau-Khalatnikov : l'atténuation tend vers zéro pour $\omega$ fini lorsque $T \rightarrow T_{\mathrm{c}}$. Ce n'est pas sans rappeler ce qui a été observé dans les phases basses températures de l'hélium [14] ou d'un antiferromagnétique [15] comme $\mathrm{MnF}_{2}$. Mais dans ces deux derniers cas, le courant (ou la déformation) était couplé linéairement au paramètre d'ordre. Ici, au contraire, le couplage avec $|\psi|$ est anharmonique. 


\section{Bibliographie}

[1] De Gennes, P. G., Solid State Commun. 10 (1972) 753

[2] Brochard, F. et Jähnig, F., J. Physique 35 (1974) 301.

[3] Cheung, L., Meyer, R. B. and Gruler, H., Phys. Rev. Lett 31 (1973) 349.

[4] Delaye, M., Ribotta, R. and Durand, G., Phys. Rev. Lett. 31 (1973) 443.

[5] LÉger, L., Phys. Lett. A 44 (1973) 535.

[6] Cladis, P. E., Phys. Rev. Lett. 31 (1973) 1200

[7] Cheung, L. and Meyer, R. B., Phys. Lett. A 43 (1973) 261.

[8] Hardouin, F., Achard, M. F., Gasparoux, H., Solid State Commun. 14 (1974) 453.
[9] Huang, C. C., Pindak, R. S., Flanders, P. J., and Ho, J. T., Phys. Rev. Lett. 33 (1974) 400.

[10] LÉger, L., Preprint.

[11] Salin, D., Smith, I. W. et Durand, G., J. Physique Lett. 35 (1974) L-165.

[12] BACRI, J. C., J. Physique Lett. 35 (1974) L-141.

[13] BACRI, J. C., 5e Conf. Internat. sur les Cristaux Liquides, Stockholm J. Physique Colloq. 36 (1975) C1-123.

[14] Rudnick, I., Phys. Rev. Lett. 25 (1970) 276.

[15] Bachellerie, A., Joffrin, J. et Levelut, A., Phys. Rev. Lett. 30 (1973) 617. 